ESAIM: M2AN 49 (2015) 257-273

DOI: $10.1051 / \mathrm{m} 2 \mathrm{an} / 2014029$
ESAIM: Mathematical Modelling and Numerical Analysis

www.esaim-m2an.org

\title{
AN ANALYSIS OF FENG'S AND OTHER SYMMETRIC LOCAL ABSORBING BOUNDARY CONDITIONS
}

\author{
Kersten Schmidt ${ }^{1}$ And Christian Heier ${ }^{1}$
}

\begin{abstract}
With symmetric local absorbing boundary conditions for the Helmholtz equation scattering problems can be solved on a truncated domain, where the outgoing radiation condition is approximated by a Dirichlet-to-Neumann map with higher tangential derivatives on its outer boundary. Feng's conditions are symmetric local absorbing boundary conditions, which are based on an asymptotic expansion of the coefficients of the exact Dirichlet-to-Neumann map for large radia of the circular outer boundary. In this article we analyse the well-posedness of variational formulations with symmetric local absorbing boundary conditions in general and show how the modelling error introduced by Feng's conditions depends on the radius of the truncated domain.
\end{abstract}

Mathematics Subject Classification. 65N30, 35J25, 78M30.

Received September 24, 2013. Revised April 8, 2014.

Published online January 16, 2015.

\section{INTRODUCTION}

We consider the scattering of an incoming time-harmonic wave (time convention $\mathrm{e}^{-\mathrm{i} \omega t}$ ) in two dimensions by a bounded object, which is modelled by the Helmholtz equation with Sommerfeld radiation condition (see e.g., [21] Chap. 1, [17] Chap. 3)

$$
\begin{array}{rlrl}
-\Delta u(x)-\kappa^{2}(x) u(x) & =0, & \text { in } & \mathbb{R}^{2} \backslash \Omega_{c} \\
\partial_{n} u(x)=0, & \text { on } & \partial \Omega_{c}, \\
u^{\mathrm{sc}}:=u-u^{\text {inc }} \text { is outgoing, }, & &
\end{array}
$$

where $\Omega_{c}$ is a bounded subdomain corresponding to a rigid body in acoustic scattering, to a perfectly electric conductor for the transverse magnetic (TM) mode and a perfectly magnetic conductor for the transverse electric (TE) mode in electromagnetic scattering. Furthermore, the possibly complex wave-number $\kappa \in L_{\text {loc }}^{\infty}\left(\mathbb{R}^{2}\right)$ has a real constant value $k>0$ outside a disk of radius $R_{C}>0$, i.e., $\kappa(x)=k$ for $|x|>R_{C}$, and $u^{\text {inc }} \in C^{\infty}\left(\mathbb{R}^{2}\right)$. The case that $\Omega_{c}$ is empty corresponds to the electromagnetic scattering by dielectric or plasmonic objects, which is the bounded subdomain where $\kappa \neq k$. We choose the Neumann boundary condition on $\partial \Omega_{c}$ only for simplicity, the analysis in this article can be simply extended to other boundary conditions including impedance boundary conditions.

\footnotetext{
Keywords and phrases. Absorbing boundary conditions, Feng's conditions, error analysis.

1 Research Center MATHEON, TU Berlin, 10623 Berlin, Germany. kersten.schmidt@math.tu-berlin.de
} 
Several local absorbing boundary conditions (ABCs) have been proposed, that are applied on the outer boundary $\Gamma$ of a truncated domain $\Omega$, which covers fully the scattering object. The ABCs applied to the scattered field $u^{\mathrm{sc}}$ on $\Gamma$ shall be mainly transparent only to an outwards radiation, why they are called transparent boundary conditions, and shall have almost no spurious reflection, why they are synonymously called nonreflecting boundary conditions. See e.g., Givoli [16] for an overview of ABCs. In general, these local ABCs are derived using an ansatz with separation of variables in a local coordinate system, which makes it necessary to use simple shapes like circles or ellipses for the artificial boundary $\Gamma$. Using the (outwards) normal derivative $\partial_{n}$ and tangential derivatives $\partial_{\tau}$ on $\Gamma$ the symmetric local absorbing boundary conditions were introduced in equation 3.14 of [19] as

$$
\left(\partial_{n} u_{J}^{\mathrm{s} c}\right)(x)+\sum_{j=0}^{J}(-1)^{j} a_{j}(k)\left(\partial_{\tau}^{2 j} u_{J}^{\mathrm{s} c}\right)(x)=0, \quad \text { on } \quad \Gamma,
$$

where $J \in \mathbb{N}_{0}$ corresponds to the order of the highest tangential derivative and $a_{j}(k)$ are coefficients depending on the choice of the boundary condition. The following ABCs belong to the class of symmetric local ABCs: Feng's conditions to any order [13], those by Givoli, Patlashenko and Keller [19] to any order as well as the first two Bayliss-Gunzburger-Turkel (BGT) conditions [5] and the first two Engquist-Majda conditions [10]. For $J=1$ these conditions are also known as Wentzell conditions $[3,9,11,12,29,30]$, see also [6] for the Laplacian and $a_{1}<0$. The above mentioned local ABC are constructed to have a convergence of the modelling error, i.e., the difference to the solution with exact Sommerfeld radiation condition, either in the order $J$, in the radius $R$ or in both. Those convergence results have been mostly observed only numerically. There is a rigorous proof for the convergence in $R$ for the Poisson problem in $2 \mathrm{D}(\kappa=0)$ and Dirichlet boundary condition on $\partial \Omega_{c}$ by Bao and Han [4] (see also [20]). To our knowledge there is no rigorous proof for convergence in $R$ for any ABC for the Helmholtz equations.

Symmetric local ABCs are well suited to be incorporated into a variational formulation, where auxiliary unknowns are not needed. In contrast to the symmetric local ABCs, the BGT conditions and Engquist-Majda conditions of order three and higher involve the inverse of a partial differential operator. In [19] the possibility that the formulation for the indefinite Helmholtz equation with symmetric local ABCs might be instable was noted, but it was not analysed further.

As the symmetric local ABCs involve high-order operators on the boundary $\Gamma$, the variational formulation requires more regularity of the trace of trial and test functions on $\Gamma$ than classical formulations used to solve Helmholtz equations. Together with a classical $C^{0}$ continuous finite element (FEM) discretisation [7] these local ABCs can be used directly with an approach adopted from discontinuous Galerkin FEM, see [27], or by using trial and test functions with $C^{(J-1)}$-continuity along $\Gamma[18,19]$ (for the latter see a combined proof of discretisation and modelling error in [4]).

Feng's conditions are derived for a circular boundary $\Gamma$ of radius $R$, where the coefficients $a_{j}$ arise from an asymptotic expansion of the coefficients of the exact Dirichlet-to-Neumann boundary conditions ([21], Eq. (3.2.4)) for large $R$. This article is dedicated to the analysis of the well-posedness of the symmetric local ABCs, the regularity of the solution (both in Sect. 2) as well as the modelling error for Feng's conditions in dependence of $R$ (Sect. 3). Numerical experiments in Section 4 confirm that the bounds on the error are sharp.

\section{SyMmETRIC LOCAL ABSORBING BOUNDARY CONDITIONS}

In this section, we recall the expression of Feng local absorbing boundary conditions in terms of differential operators acting on the outer boundary $\Gamma$ of the computational domain $\Omega$ (see Fig. 1). The outer boundary $\Gamma$ is assumed to be $C^{\infty}$ such that continuous (outer) normal and tangential unit vectors $e_{n}$ can be defined. Then, we define the normal and tangential derivatives as

$$
\left(\partial_{n} v\right)(x):=\nabla v(x) \cdot e_{n}(x), \quad\left(\partial_{\tau} v\right)(x):=\nabla v(x) \cdot e_{\tau}(x),
$$



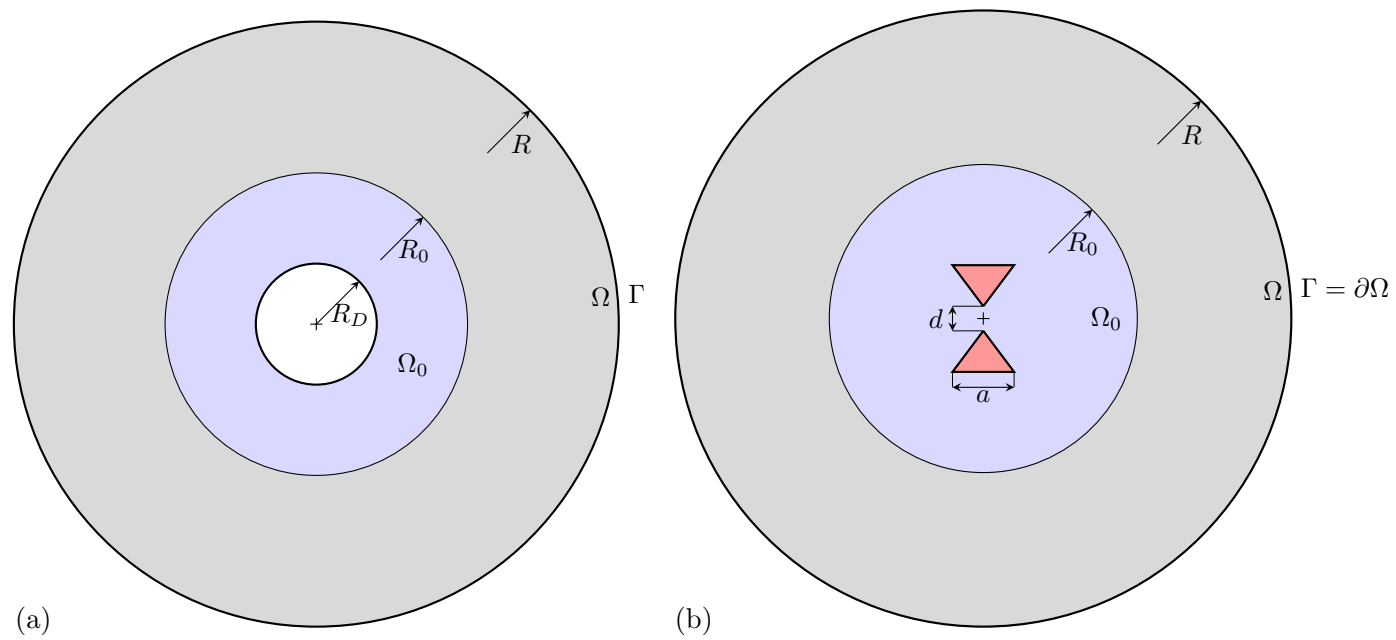

FIgURE 1 . The geometrical setting for the (a) acoustic scattering on a rigid cylinder of radius $R_{D}=1$, and (b) for the electromagnetic scatting on two cylinders with equilateral triangles as cross-section of length $a=1.05$ and distance $d=0.25$. The error is measured in $\Omega_{0}$, the (a) ring or (b) disk of fixed outer radius $R_{0}$ (blue), while the (outer) radius $R$ of $\Omega$ varies for the convergence analysis of the modelling error of the local DtN boundary conditions.

where for a circle of radius $R>R_{C}$ with origin as mid-point we have $e_{n}(x)=x /|x|$ and $e_{\tau}(x)=\left(-x_{2}, x_{1}\right)^{\top} /|x|$ and $\left(\partial_{n} v\right)(x)=\partial_{r} v(x(r, \theta)),\left(\partial_{\tau} v\right)(x)=R^{-1} \partial_{\theta} v(x(r, \theta))$.

\subsection{Definition of symmetric local absorbing boundary conditions}

We write the Helmholtz equation with symmetric local absorbing boundary conditions on $\Gamma$ (see e.g. [19], Eq. (3.14)) as

$$
\begin{aligned}
& -\Delta u_{J}(x)-\kappa^{2}(x) u_{J}(x)=0, \quad \text { in } \quad \Omega \\
& \partial_{n} u_{J}(x)=0, \quad \text { on } \quad \partial \Omega \backslash \Gamma, \\
& u_{J}(x)-u_{J}^{\mathrm{sc}}(x)-u^{\mathrm{inc}}(x)=0, \quad \text { in } \quad \Omega \\
& \left(\partial_{r} u_{J}^{\mathrm{sc}}\right)(x)+\sum_{j=0}^{J}(-1)^{j} a_{j}(k, R)\left(\partial_{\tau}^{2 j} u_{J}^{\mathrm{sc}}\right)(x)=0, \quad \text { on } \quad \Gamma \text {. }
\end{aligned}
$$

In a forthcoming article [27] it will be shown, that Feng's local absorbing boundary conditions can be discretised without introducing any auxiliary variables. Moreover, the resulting linear system is symmetric, which limits the computational cost of the inversion step.

For the conditions by Feng of order $N \in \mathbb{N}_{0}$, abbreviated by Feng- $N$, the order of the highest derivative $J=\lfloor N / 2\rfloor$, where the coefficients $a_{j}(k, R)$ take the form

$$
a_{j}(k, R)=k^{1-2 j} \alpha_{j}^{N}\left((k R)^{-1}\right) .
$$

Here, $\alpha_{j}^{N}$ are polynomials of order $N-2 j$, which are given for $N=0, \ldots, 5$ in Table 1 . Note, that Feng-0 is also known as the "Sommerfeld-like" condition ([17], Chap. 3) and Feng-1 is equivalent to the BGT-1, the first condition by Bayliss, Gunzberger and Turkel [5]. 
TABle 1. Polynomials $\alpha_{j}^{N}$ in (2.2) for Feng's conditions for order $N=0, \ldots, 5$, where $y=(k R)^{-1}$.

\begin{tabular}{|c|c|c|c|}
\hline$N$ & $\alpha_{0}^{N}(y)$ & $\alpha_{1}^{N}(y)$ & $\alpha_{2}^{N}(y)$ \\
\hline 0 & $-\mathrm{i}$ & & \\
\hline 1 & $-\mathrm{i}+\frac{y}{2}$ & & \\
\hline 2 & $-\mathrm{i}+\frac{y}{2}-\frac{\mathrm{i}}{8} y^{2}$ & $\frac{\mathrm{i}}{2}$ & \\
\hline 3 & $-\mathrm{i}+\frac{y}{2}-\frac{\mathrm{i}}{8} y^{2}-\frac{y^{3}}{8}$ & $\frac{\mathrm{i}}{2}-\frac{y}{2}$ & \\
\hline 4 & $-\mathrm{i}+\frac{y}{2}-\frac{\mathrm{i}}{8} y^{2}-\frac{y^{3}}{8}+\frac{25 \mathrm{i}}{128} y^{4}$ & $\frac{\mathrm{i}}{2}-\frac{y}{2}-\frac{13 \mathrm{i}}{16} y^{2}$ & $\frac{\mathrm{i}}{8}$ \\
\hline 5 & $-\mathrm{i}+\frac{y}{2}-\frac{\mathrm{i}}{8} y^{2}-\frac{y^{3}}{8}+\frac{25 \mathrm{i}}{128} y^{4}+\frac{13}{32} y^{5}$ & $\frac{\mathrm{i}}{2}-\frac{y}{2}-\frac{13 \mathrm{i}}{16} y^{2}-\frac{7}{4} y^{3}$ & $\frac{\mathrm{i}}{8}+\frac{y}{2}$ \\
\hline
\end{tabular}

\subsection{Variational formulation}

For the variational formulation we define the Sobolev spaces

$$
V_{0}:=H^{1}(\Omega), \quad V_{J}:=H^{1}(\Omega) \cap H^{J}(\Gamma), \quad J>0,
$$

where $V_{0}$ is equipped with the usual $H^{1}(\Omega)$-norm and $V_{J}$ with the norm defined by

$$
\|v\|_{V_{J}}^{2}:=\|v\|_{H^{1}(\Omega)}^{2}+\sum_{j=1}^{J}|v|_{H^{j}(\Gamma)}^{2} .
$$

Then, we can state the variational formulation of (2.1): Seek $u_{J} \in V_{J}$ such that

$$
\mathrm{a}_{J}\left(u_{J}, v\right)=\left\langle f_{J}, v\right\rangle, \quad \forall v \in V_{J},
$$

where

$$
\begin{aligned}
\mathrm{a}_{J}(u, v) & :=\int_{\Omega}\left(\nabla u \cdot \nabla \bar{v}-\kappa^{2} u \bar{v}\right) \mathrm{d} x+\sum_{j=0}^{J} a_{j} \int_{\Gamma} \partial_{\tau}^{j} u \partial_{\tau}^{j} \bar{v} \mathrm{~d} \sigma(x), \\
\left\langle f_{J}, v\right\rangle & :=\int_{\Gamma}\left(\partial_{r} u^{\mathrm{inc}} \bar{v}+\sum_{j=0}^{J} a_{j} \partial_{\tau}^{j} u^{\mathrm{inc}} \partial_{\tau}^{j} \bar{v}\right) \mathrm{d} \sigma(x)
\end{aligned}
$$

Lemma 2.1. Let $\left|\operatorname{Im}\left(a_{J}\right)\right|>0$ or $\operatorname{Re}\left(a_{J}\right)>0$. Then, the bilinear form $\mathrm{a}_{0, J}$ defined by

$$
\begin{aligned}
\mathrm{a}_{0, J}(u, v):= & \int_{\Omega}(\nabla u \cdot \nabla \bar{v}+u \bar{v}) \mathrm{d} x \\
& +\sum_{j=0}^{J-1} \int_{\Gamma} \partial_{\tau}^{j} u \partial_{\tau}^{j} \bar{v} \mathrm{~d} \sigma(x)+a_{J} \int_{\Gamma} \partial_{\tau}^{J} u \partial_{\tau}^{J} \bar{v} \mathrm{~d} \sigma(x),
\end{aligned}
$$

is $V_{J}$-elliptic.

Proof. By the assumption on $a_{J}$ there exists a constant $\theta \in\left(-\frac{\pi}{2}, \frac{\pi}{2}\right)$ such that $a_{J}=\left|a_{J}\right| \mathrm{e}^{2 \mathrm{i} \theta}$. Then,

$$
\operatorname{Re}\left(\mathrm{e}^{-\mathrm{i} \theta} \mathrm{a}_{0, J}(v, v)\right)=\cos (\theta)\left(\|v\|_{V_{J-1}}^{2}+\|v\|_{L^{2}(\Gamma)}^{2}+\left|a_{J} \| v\right|_{H^{J}(\Gamma)}^{2}\right) \geq \gamma\|v\|_{V_{J}}^{2},
$$

where $\gamma:=\cos (\theta) \min \left(1,\left|a_{J}\right|\right)>0$ by the assumption on $a_{J}$. This is exactly the statement of the lemma. (see [26], Eq. (2.43)). 
Corollary 2.2. For Feng's conditions of order $N=0, \ldots, 5$ the bilinear forms $\mathrm{a}_{0, J}, J=\lfloor N / 2\rfloor$ are $V_{J}$-elliptic. Proof. For any $y \in \mathbb{R}^{+}$it holds $\operatorname{Im} \alpha_{0}^{N}(y)=-1$ for $N=0,1, \operatorname{Im} \alpha_{1}^{N}(y)=\frac{1}{2}$ for $N=2,3$ and $\operatorname{Im} \alpha_{2}^{N}(y)=\frac{1}{8}$ for $N=4,5$. Applying Lemma 2.1 completes the proof.

Theorem 2.3. Let the assumption of Lemma 2.1 be satisfied and let zero be the only solution of (2.3) with $u^{\mathrm{inc}}=0$ (and so $f_{J}=0$ ). Then, for any $f_{J} \in V_{J}^{\prime}$ there exists a unique solution $u_{J} \in V_{J}$ of $(2.3)$ and a constant $C_{J}>0$ such that

$$
\left\|u_{J}\right\|_{V_{J}} \leq C_{J}\left\|f_{J}\right\|_{V_{J}^{\prime}}
$$

Proof. By Lemma 2.1 the bilinear form $\mathrm{a}_{0, J}$ is $V_{J}$-elliptic and so the associated operators $A_{0, J}$ are isomorphism in $V_{J}$. We define the Sobolev spaces

$$
W_{0}:=L^{2}(\Omega), \quad W_{J}:=L^{2}(\Omega) \cap H^{J-1}(\Gamma), \quad J>0,
$$

and the Rellich-Kondrachov compactness Theorem ([2], Chap. 6) implies that the embedding $V_{J} \subset \subset W_{J}$ is compact. Now, we define the bilinear forms

$$
\mathrm{k}_{J}(u, v):=-\int_{\Omega}\left(\kappa^{2}+1\right) u \bar{v} \mathrm{~d} x+\sum_{j=0}^{J-1}\left(a_{j}-1\right) \int_{\Gamma} \partial_{\tau}^{j} u \partial_{\tau}^{j} \bar{v} \mathrm{~d} \sigma(x), \quad J>0,
$$

and their associated operators $K_{J}$ are compact perturbations of $A_{0, J}$. Hence, the operators $A_{0, J}+K_{J}$ associated to the bilinear forms $\mathrm{a}_{J}=\mathrm{a}_{0, J}+\mathrm{k}_{J}$ are Fredholm with index 0 and by the Fredholm alternative ([26], Sect. 2.1.4) the uniqueness of a solution of (2.3) implies its existence and continuous dependence on the right hand side. As uniqueness holds by assumption we assert the statement of the theorem.

Lemma 2.4. Let $\inf _{x \in \Omega} \operatorname{Im}\left(\kappa^{2}\right) \geq 0, \operatorname{Im}\left(a_{0}\right)<0$ and $\operatorname{Im}\left(a_{j}\right) \leq 0,1 \leq j \leq J$. Then, the variational formulation (2.3) has a unique solution $u_{J} \in H^{1}(\Omega)$.

Proof. By Theorem 2.3 it is enough to show uniqueness, for which we assume $u^{\mathrm{inc}}=0$ and so $u_{J}=u_{J}^{\mathrm{sc}}$ in the following. Then, inserting $v=u_{J}$ into (2.3) and taking the imaginary part we obtain

$$
0=-\int_{\Omega} \operatorname{Im}\left(\kappa^{2}\right)\left|u_{J}\right|^{2} \mathrm{~d} x+\sum_{j=0}^{J} \operatorname{Im}\left(a_{j}\right)\left|u_{J}\right|_{H^{j}(\Gamma)}^{2} .
$$

By the assumption of the lemma the right hand side is a sum of non-positive terms and the equality holds only if they vanish all to zero. As the imaginary part of $a_{0}$ is negative we can assert that $\left\|u_{J}\right\|_{L^{2}(\Gamma)}=0$ and so $u_{J}=0$ on $\Gamma$. In view of $(2.1 \mathrm{~d})$ we have $\partial_{r} u_{J}=0$ on $\Gamma$. Now, we can extend $u_{J}$ in a exterior neighbourhood of $\Gamma$. Let $\widetilde{\Omega}$ be a Lipschitz domain such that $\Omega \subsetneq \widetilde{\Omega}$. In $\widetilde{\Omega} \backslash \Omega$ we define $u_{J}=0$ such that $u_{J}$ and $\partial_{r} u_{J}$ are continuous over $\Gamma$. Furthermore, we let $\kappa=k$ in $\widetilde{\Omega} \backslash \Omega$. In this way $u_{J}$ is solution of

$$
-\Delta u_{J}(x)-\kappa^{2}(x) u_{J}(x)=0, \quad \text { in } \widetilde{\Omega} .
$$

If $\widetilde{\Omega}$ is simply connected we can conclude with the unique continuation principle ([23], Chap. 4.3) that $u_{J}=0$ in $\Omega$ as well. If $\widetilde{\Omega}$ is not simply connected we can find a finite covering of overlapping simply connected panels $\Omega_{k}$, where the ordering is such that $\Omega_{0} \cap \widetilde{\Omega} \neq \emptyset$ and $\Omega_{k} \cap \Omega_{k+1} \neq \emptyset$ for all $k$. Then, we apply the unique continuation principle after each other to $\Omega_{k}, k=0,1, \ldots$ and conclude eventually in $u_{J}=0$ in $\Omega$. This completes the proof.

Corollary 2.5. Let $\inf _{x \in \Omega} \operatorname{Im}\left(\kappa^{2}\right) \geq 0$. Then, the variational formulation (2.3) with Feng's conditions of order $N=0,1$ has a unique solution in $H^{1}(\Omega)$.

The related eigenvalue problem is a perturbation of a linear one in $\omega^{2}$, and we can use for $N>1$ the following. 
Lemma 2.6. Let the assumption of Lemma 2.1 be satisfied, let $a_{j}(k), j=0,1, \ldots, J, J>0$ be analytic for $k \in \mathbb{R}^{+}$, where there exist numbers $a_{j, \ell} \in \mathbb{C}$ such that $a_{j}(k)=k^{1-2 j} \sum_{\ell=0}^{2(J-j)} a_{j, \ell} k^{-\ell}, \operatorname{Re}\left(a_{0,1}\right)>0$ or $\left|\operatorname{Im}\left(a_{0,1}\right)\right|>0$, and $\operatorname{Re}\left(a_{J}(k)\right)>0$ or $\left|\operatorname{Im}\left(a_{J}(k)\right)\right|>0$ for any $k \in \mathbb{R}^{+}$. Furthermore, let $c^{-1} \in L_{\mathrm{loc}}^{\infty}\left(\mathbb{R}^{2}\right)$ be fixed with $c(x)=c_{0}>0$ for $|x|>R_{C}$ and $\kappa(x)=\omega / c(x)$ with the frequency $\omega \in \mathbb{R}^{+}$. Then, (2.3) has a unique solution except for a possibly infinite set of isolated values $\omega$, the spurious eigenfrequencies, which accumulates only at infinity.

Proof. We regard the eigenvalue problem related to (2.3) in a fixed point form. For this we define

$$
\begin{aligned}
\mathrm{a}_{0, J}(u, v ; \widetilde{\omega}) & :=\int_{\Omega} \nabla u \cdot \nabla \bar{v}+u \bar{v} \mathrm{~d} x+\sum_{j=0}^{J-1} \int_{\Gamma} \partial_{\tau}^{j} u \partial_{\tau}^{j} \bar{v} \mathrm{~d} \sigma(x)+a_{J}\left(\widetilde{\omega} / c_{0}\right) \int_{\Gamma} \partial_{\tau}^{J} u \partial_{\tau}^{J} \bar{v} \mathrm{~d} \sigma(x), \\
\mathrm{k}_{J}(u, v ; \widetilde{\omega}) & :=-\int_{\Omega}\left(\frac{\widetilde{\omega}^{2}}{c^{2}(x)}+1\right) u \bar{v} \mathrm{~d} x+\sum_{j=0}^{J-1}\left(a_{j}\left(\widetilde{\omega} / c_{0}\right)-1\right) \int_{\Gamma} \partial_{\tau}^{J} u \partial_{\tau}^{J} \bar{v} \mathrm{~d} \sigma(x),
\end{aligned}
$$

where $\widetilde{\omega} \in \mathbb{R}^{+}$acts as a parameter. Hence, we can rewrite the eigenvalue problem (2.3) with $f_{J}=0$ as: Find $\left(u_{J}, \omega\right) \in V_{J} \times \mathbb{R}^{+}$such that

$$
\mathrm{a}_{0, J}\left(u_{J}, v ; \omega\right)+\mathrm{k}_{J}\left(u_{J}, v ; \omega\right)=0, \quad \forall v \in V_{J}
$$

In its fix-point form

$$
\mathrm{a}_{0, J}\left(u_{J}, v ; \widetilde{\omega}\right)+\frac{\omega^{2}}{\widetilde{\omega}^{2}} \mathrm{k}_{J}\left(u_{J}, v ; \widetilde{\omega}\right)=0,
$$

it is a linear eigenvalue problem in $\omega^{2}$. The operator valued function $A_{0, J}(\widetilde{\omega})$ related to $\mathrm{a}_{0, J}(\cdot, \cdot ; \widetilde{\omega})$ is by assumption on $a_{J}$ an isomorphism and the operator valued function $K_{J}(\widetilde{\omega})$ related to $\mathrm{k}_{J}(\cdot, \cdot ; \widetilde{\omega})$ is compact, both for any $\widetilde{\omega} \in \mathbb{R}^{+}$and in the limit $\widetilde{\omega} \rightarrow \infty$. By the Fredholm-Riesz-Schauder theory ([26], Sect. 2.1.4) we have for each $\widetilde{\omega} \in \mathbb{R}^{+}$a countable set of frequencies $\omega_{m}(\widetilde{\omega}) \in \mathbb{C}, m \in \mathbb{N}$, which accumulates only at infinity. As the eigenvalue problem depends for $\widetilde{\omega}>0$ analytically on $\widetilde{\omega}$, we can find an ordering such that $\omega_{m}(\widetilde{\omega})$ are analytic functions. The original problem has spurious eigenfrequencies for $\omega=\widetilde{\omega}$, this is where a curve $\omega_{m}(\widetilde{\omega})$ meets the linear curve $\omega_{c}(\widetilde{\omega})=\widetilde{\omega}$. Due to its analyticity a curve $\omega_{m}(\widetilde{\omega})$ can only meet $\omega_{c}(\widetilde{\omega})$ in a countable set of values $\widetilde{\omega}$ or $\omega_{m}(\widetilde{\omega})=\omega_{c}(\widetilde{\omega})$ for all $\widetilde{\omega} \in \mathbb{R}^{+}$. If the latter would be true, then a (non-trivial) eigenfunction $u=u_{J}$ would exists in the limit $\widetilde{\omega}=\omega \rightarrow 0$. We will show that this is not true.

Multiplying (2.5) with $v=u$, and using for a simpler writing the abbreviation $k=\omega / c_{0}$ we find

$$
|u|_{H^{1}(\Omega)}^{2}-k^{2} c_{0}^{2}\left\langle c^{-2} u, u\right\rangle+\sum_{j=0}^{J} k^{1-2 j} \sum_{\ell=0}^{2(J-j)} a_{j, \ell} k^{-\ell}|u|_{H^{j}(\Gamma)}=0 .
$$

We normalise the eigenfunction $u$, such that $\|u\|_{V_{J}}=1$, and so all the terms $|u|_{H^{1}(\Omega)}^{2}, c_{0}^{2}\left\langle c^{-2} u, u\right\rangle,|u|_{H^{j}(\Gamma)}$, $j=0, \ldots, J$ are bounded. In (2.7) there are terms with negative powers in $k$, i.e., these terms may tend to infinity for $k \rightarrow 0$. If $u$ is eigensolution of (2.7) for $k \rightarrow 0$, then all terms in $k^{-m}, m=1, \ldots, 2 J-1$ have to be zero in sum, i.e., for $m=1, \ldots, 2 J-1$ it holds

$$
\sum_{j=0}^{\left\lfloor\frac{\left.\frac{m+1}{2}\right\rfloor}{2}\right.} a_{j, m+1-2 j}|u|_{H^{j}(\Gamma)}^{2}=0 .
$$

We can regard (2.8) as system of $(2 J-1) \cdot 2$ linear equations (real and complex part) for $J+1$ real variables $|u|_{H^{j}(\Gamma)}^{2}, j=0, \ldots, J$. If this system has no solution except the trivial one, $u=0$, then our assumption $\|u\|_{V_{J}}=1$ 
was false. Otherwise, (2.7) simplifies in the limit $k \rightarrow 0$ to

$$
|u|_{H^{1}(\Omega)}^{2}+a_{0,1}\|u\|_{L^{2}(\Gamma)}^{2}=0,
$$

and with the assumption on $a_{0,1}$ it follows similarly to the proof of Lemma 2.1 that there is only a trivial solution $u=0$. Hence, we have $\omega_{m}(\widetilde{\omega})=\widetilde{\omega}$ only for isolated values of $\widetilde{\omega}$, and the proof is complete.

In the limit $R \rightarrow \infty$ Feng's conditions of any order $N$ converge to that of order 0 , for which the problem is well-posed for all frequencies except for $\omega=0$, and so Feng's conditions provide a unique solution if the domain is large enough. This is formulated in the following lemma, whose proof will be given in Appendix.

Lemma 2.7. Let $\inf _{x \in \Omega} \operatorname{Im}\left(\kappa^{2}\right) \geq 0, \lim _{R \rightarrow \infty} a_{0}=-\mathrm{i} k, \lim _{R \rightarrow \infty} R^{-2 j}\left|\operatorname{Im} a_{j}\right|=0, j=1, \ldots, J, J>0$. Then, there exists a number $R_{\text {unique }}>0$ such that for any $R>R_{\text {unique }}$ the variational formulation (2.3) has a unique solution in $H^{1}(\Omega)$.

As the outer boundary $\Gamma$ is analytic and the coefficients $\alpha_{j}$ are scalar, hence analytic, we can assert the following

Lemma 2.8. Let $u_{J} \in V_{J}$ be solution of (2.3). Then, $u_{J} \in H^{j}(\Gamma)$ for any $j \in \mathbb{N}$.

Proof. As the incoming field $u^{\text {inc }}$ is assumed to be $C^{\infty}$, it is enough to show the regularity statement for the scattered field $u_{J}^{\mathrm{sc}} \in H^{J}(\Gamma)$. The proof is by induction in $k \in \mathbb{N}_{0}$, in particular we show that $u_{J}^{\mathrm{sc}} \in H^{J+k}(\Gamma)$ implies $u_{J}^{\mathrm{sc}} \in H^{J+k+2}(\Gamma)$. By the elliptic regularity theory ([24], Thm. 4.18) we have $u_{J}^{\mathrm{sc}} \in H^{J+1 / 2+k}\left(\Omega_{\Gamma}\right)$ for a neighbourhood $\Omega_{\Gamma} \subset \Omega$ of $\Gamma$ and so $\partial_{r} u_{J}^{\mathrm{sc}} \in H^{J-1 / 2+k}(\Gamma)$. We rewrite (2.1d) as

$$
(-1)^{J} a_{J} \partial_{\tau}^{2 J} u_{J}^{\mathrm{sc}}=\partial_{r} u_{J}^{\mathrm{sc}}-\sum_{j=0}^{J-1}(-1)^{j} a_{j} \partial_{\tau}^{2 j} u_{J}^{\mathrm{sc}} \in H^{2-J+k}(\Gamma),
$$

and so by the elliptic shift theorem $u_{J}^{\mathrm{sc}} \in H^{J+k+2}(\Gamma)$. This completes the proof.

\section{Absorbing boundary conditions by Feng}

\subsection{Introduction}

The absorbing boundary conditions by Feng [13] origins from an asymptotic expansion of the exact Dirichletto-Neumann (DtN) boundary conditions. The exact scattered field $u^{\mathrm{sc}}:=u-u^{\text {inc }}$ fulfills

$$
\left(\partial_{r} u^{\mathrm{sc}}\right)(x)=\sum_{n \in \mathbb{Z}} \lambda_{n}(k, R) \mathrm{e}^{\mathrm{i} n \theta(x)} \widehat{u}_{n}^{\mathrm{sc}}
$$

where $\widehat{u}_{n}^{\mathrm{sc}}=\frac{1}{2 \pi R} \int_{\Gamma} u^{\mathrm{sc}}(x) \mathrm{e}^{-\mathrm{i} n \theta(x)} \mathrm{d} \sigma(x)$ is the $n$th Fourier component of the Dirichlet trace $u^{\mathrm{sc}}$ on $\Gamma$, and with the Hankel functions $H_{n}^{(1)}$, see [1], page 363ff, the DtN coefficients are defined by

$$
\lambda_{n}(k, R):=k \frac{H_{n}^{(1) \prime}(k R)}{H_{n}^{(1)}(k R)} .
$$

Since the $n$th Hankel function tends for $R \rightarrow \infty$ to $\sqrt{\frac{2}{\pi k R}} \mathrm{e}^{\mathrm{i}(k R-n \pi / 2-\pi / 4)}$ the scattered field satisfies the Sommerfeld radiation condition ([21], Chap. 1). The exact DtN boundary conditions (3.1) are non-local as they include within $\widehat{u}_{n}^{\mathrm{sc}}$ integrals of the Dirichlet trace. 
In the following we are going to derive Feng's conditions for the sake of completeness and write them for the error analysis in the same form as (3.1), but with different coefficients. We start with the asymptotic expansion of the Hankel functions for large arguments

$$
H_{n}^{(1)}(k R)=\sqrt{\frac{2}{\pi k R}} \mathrm{e}^{\mathrm{i}\left(k R-\left(\frac{n}{2}+\frac{1}{4}\right) \pi\right)} \sum_{m=0}^{\infty}\left(\frac{\mathrm{i}}{2 k R}\right)^{m} \frac{p_{m}\left(n^{2}\right)}{m !},
$$

where $p_{m}(\mu)$ is the polynomial of degree $m$ in $n^{2}([1], 9.2 .7-10)$ given by

$$
p_{m}\left(n^{2}\right)=\underbrace{\left(4 n^{2}-1\right)\left(4 n^{2}-3\right) \ldots\left(4 n^{2}-2 m-1\right)}_{m \text { terms }} .
$$

This asymptotic expansion is used to derive an asymptotic expansion of the coefficient $\lambda_{n}(k, R)$ of the exact DtN boundary

$$
\lambda_{n}(k, R) \sim \mathrm{i} k \sum_{m=0}^{\infty}\left(\frac{\mathrm{i}}{2 k R}\right)^{m} a_{m}\left(n^{2}\right),
$$

where " " indicates that it may not be a convergent series for $m \rightarrow \infty$, but the equality holds asymptotically for $R \rightarrow \infty$. More precisely, for any $n$ there exists a monotonously increasing function $R_{\min }(n)$ such that for $R>R_{\min }(n)$ the series in (3.2) converges to $\lambda_{n}(k, R)$. The functions $a_{m}$ in (3.2) are polynomials of degree $\left\lfloor\frac{m}{2}\right\rfloor$ which are defined recursively by

$$
a_{0}\left(n^{2}\right)=a_{1}\left(n^{2}\right)=1, \quad a_{2}\left(n^{2}\right)=2 n^{2}-\frac{1}{2}, \quad a_{k}\left(n^{2}\right)=(2 k-2)(n, k-1)-\sum_{\ell=2}^{k-1} a_{\ell}\left(n^{2}\right)(n, k-\ell),
$$

where the function $(n, k)$ is given by $(n, k)=\frac{1}{k !} \prod_{\ell=1}^{k}\left(n^{2}-\left(\frac{2 \ell-1}{2}\right)^{2}\right)$.

In the expression of the exact DtN boundary condition (3.1) the coefficient $\lambda_{n}(k, R)$ is multiplied by $\mathrm{e}^{\mathrm{i} n \theta}$. As $a_{m}$ are polynomials in $n^{2}$ we can assert the equality

$$
a_{m}\left(n^{2}\right) \mathrm{e}^{\mathrm{i} n \theta}=a_{m}\left(-\partial_{\theta}^{2}\right) \mathrm{e}^{\mathrm{i} n \theta} .
$$

Using this equality, inserting the asymptotic expansion $(3.2)$ of $\lambda_{n}(k, R)$ into the exact DtN boundary condition $(3.1)$ and as $a_{m}\left(-\partial_{\theta}^{2}\right)$ we obtain

$$
\begin{aligned}
\partial_{r} u^{\mathrm{sc}}(x) & \sim \mathrm{i} k \sum_{m=0}^{\infty}\left(\frac{\mathrm{i}}{2 k R}\right)^{m} a_{m}\left(-\partial_{\theta}^{2}\right) \sum_{n \in \mathbb{Z}} \widehat{u}_{n}^{\mathrm{sc} c} \mathrm{e}^{\mathrm{i} n \theta} \\
& \sim \mathrm{i} k \sum_{m=0}^{\infty}\left(\frac{\mathrm{i}}{2 k R}\right)^{m} a_{m}\left(-\partial_{\theta}^{2}\right) u^{\mathrm{sc}}(x(r, \theta)), \\
& \sim \mathrm{i} k \sum_{m=0}^{\infty}\left(\frac{\mathrm{i}}{2 k R}\right)^{m} a_{m}\left(-R^{2} \partial_{\tau}^{2}\right) u^{\mathrm{sc}}(x) .
\end{aligned}
$$

Truncating the sum at $m=N$ we obtain conditions by Feng of order $N$,

$$
\partial_{r} u_{F, N}^{\mathrm{sc}}(x)=\mathrm{i} k \sum_{m=0}^{N}\left(\frac{\mathrm{i}}{2 k R}\right)^{m} a_{m}\left(-R^{2} \partial_{\tau}^{2}\right) u_{F, N}^{\mathrm{sc}}(x)=:\left(\mathrm{F}_{N}(R) u_{F, N}^{\mathrm{sc}}\right)(x)
$$


TABLE 2. The DtN operators $\mathrm{F}_{N}(R)$ for Feng's conditions for $N=0,1, \ldots, 5$.

\begin{tabular}{l}
$\mathrm{F}_{0}(R):=\mathrm{i} k$, \\
$\mathrm{F}_{1}(R):=\mathrm{i} k-\frac{1}{2 R}$, \\
$\mathrm{F}_{2}(R):=\left(\mathrm{i} k-\frac{1}{2 R}+\frac{\mathrm{i}}{8 k R^{2}}\right)+\frac{\mathrm{i}}{2 k} \partial_{\tau}^{2}$, \\
$\mathrm{F}_{3}(R):=\left(\mathrm{i} k-\frac{1}{2 R}+\frac{\mathrm{i}}{8 k R^{2}}+\frac{1}{8 k^{2} R^{3}}\right)+\left(\frac{\mathrm{i}}{2 k}-\frac{1}{2 k^{2} R}\right) \partial_{\tau}^{2}$, \\
$\mathrm{F}_{4}(R):=\mathrm{F}_{3}(R)-\frac{25 \mathrm{i}}{128 k^{3} R^{4}}-\frac{13 \mathrm{i}}{16 k^{3} R^{2}} \partial_{\tau}^{2}-\frac{\mathrm{i}}{8 k^{3}} \partial_{\tau}^{4}$, \\
$\mathrm{F}_{5}(R):=\mathrm{F}_{4}(R)-\frac{13}{32 k^{4} R^{5}}-\frac{7}{4 k^{4} R^{3}} \partial_{\tau}^{2}-\frac{1}{2 k^{4} R} \partial_{\tau}^{4}$. \\
\hline
\end{tabular}

which compromise tangential derivatives of degree $2,4, \ldots, 2\lfloor N / 2\rfloor$, but no integrals like in (3.1), and are, hence, local. The operators $\mathrm{F}_{N}(R)$ for Feng's conditions up to order 5 are given in Table 2. For the analysis we will need an equivalent expression for (3.4)

$$
\partial_{r} u_{F, N}^{\mathrm{sc}}(x)=\sum_{n \in \mathbb{Z}} \lambda_{n}^{N}(k, R) \mathrm{e}^{\mathrm{i} n \theta(x)} \widehat{u}_{F, N}^{\mathrm{sc}}(x),
$$

with

$$
\lambda_{n}^{N}(k, R):=\mathrm{i} k \sum_{m=0}^{N}\left(\frac{\mathrm{i}}{2 k R}\right)^{m} a_{m}\left(n^{2}\right) .
$$

Comparing the definition of the exact and the approximative DtN coefficients we find that asymptotically for $k R \rightarrow \infty$

$$
\left|\lambda_{n}^{N}(k, R)-\lambda_{n}(k, R)\right| \leq C_{N}(n) k(k R)^{-(N+1)},
$$

where the constants $C_{N}(n)=\mathcal{O}_{n \rightarrow \infty}\left(n^{2\lfloor N / 2\rfloor}\right)$ do not depend on $R$ or $k$.

\subsection{Approximative "no reflection"}

Whenever an absorbing boundary condition, often alternatively called non-reflecting, is introduced, which approximate the exact outgoing behaviour of the solution, the approximative solution has an inwards radiating contribution, which can be interpreted as reflection on the artificial boundary $\Gamma$.

Therefore, the approximative solution in the part of $\Omega$ with homogeneous material, i.e., for $R_{C}<r \leq R$, can be written as sum of outwards and inwards radiating Hankel functions $H_{n}^{(1)}$ and $H_{n}^{(2)}$, respectively,

$$
u_{F, N}^{\mathrm{s} c}(x)=\sum_{n \in \mathbb{Z}}\left(a_{n}^{N} H_{n}^{(1)}(k r(x))+b_{n}^{N} H_{n}^{(2)}(k r(x))\right) \mathrm{e}^{\mathrm{i} n \theta(x)} .
$$

The coefficients $b_{n}^{N}$ correspond to the "reflected" contribution, and we may ask ourself when the ratio $b_{n}^{N} / a_{n}^{N}$ of the coefficients of the inwards and the outwards radiating contribution gets smaller.

Now, taking the normal derivative on $\Gamma$ and using the equality (3.5) we obtain

$$
\begin{aligned}
\partial_{r} u_{F, N}^{\mathrm{sc}}(x) & =\sum_{n \in \mathbb{Z}} k\left(a_{n}^{N} H_{n}^{(1) \prime}(k R)+b_{n}^{N} H_{n}^{(2) \prime}(k R)\right) \mathrm{e}^{\mathrm{i} n \theta(x)} \\
& =\sum_{n \in \mathbb{Z}} \lambda_{n}^{N}(k, R)\left(a_{n}^{N} H_{n}^{(1)}(k R)+b_{n}^{N} H_{n}^{(2)}(k R)\right) \mathrm{e}^{\mathrm{i} n \theta(x)},
\end{aligned}
$$


which is a condition to relate $a_{n}^{N}$ and $b_{n}^{N}$

$$
a_{n}^{N}\left(k H_{n}^{(1) \prime}(k R)-\lambda_{n}^{N}(k, R) H_{n}^{(1)}(k R)\right)=-b_{n}^{N}\left(k H_{n}^{(2) \prime}(k R)-\lambda_{n}^{N}(k, R) H_{n}^{(2)}(k R)\right) .
$$

Hence, we find that the ratio between the inwards and the outwards radiating contributions

$$
\begin{aligned}
\frac{b_{n}^{N}}{a_{n}^{N}} & =-\frac{k H_{n}^{(1) \prime}(k R)-\lambda_{n}^{N}(k, R) H_{n}^{(1)}(k R)}{k H_{n}^{(2) \prime}(k R)-\lambda_{n}^{N}(k, R) H_{n}^{(2)}(k R)} \\
& =\frac{\lambda_{n}^{N}(k, R)-\lambda_{n}(k, R)}{\left(H_{n}^{(1)}(k R)\right)^{-1}\left(k H_{n}^{(2) \prime}(k R)-\lambda_{n}^{N}(k, R) H_{n}^{(2)}(k R)\right)}:=-E_{n}^{N}(k, R)
\end{aligned}
$$

gets the smaller the better $\lambda_{n}^{N}(k, R)$ approximates $\lambda_{n}(k, R)$. The term $k H_{n}^{(2) \prime}(k R)-\lambda_{n}^{N}(k, R) H_{n}^{(2)}(k R)$ in the denominator in the expression of the "non-reflectance efficiency" $E_{n}^{N}(k, R)$ in (3.9) would tend to zero for $R \rightarrow \infty$ if Feng's conditions would be approximations to an exact "inwards radiation condition", which they are simply not. Therefore, the denominator tends to a constant expresses how accurate inwards radiating waves satisfy Feng's conditions. Hence, using (3.6) we can bound the "non-reflectance efficiency" asymptotically for $R \rightarrow \infty$ by

$$
\left|E_{n}^{N}(k, R)\right| \leq C_{N}(n)(k R)^{-(N+1)},
$$

where $C_{N}(n)$ are constants independent of $R$ and $k$. As $\lim _{n \rightarrow \infty}\left|E_{n}^{N}(k, R)\right|=1$ we have $C_{N}(n)=\mathcal{O}_{n \rightarrow \infty}(1)$.

\subsection{Error analysis}

By the truncation of the asymptotic expansion (3.3) of the exact DtN boundary condition at order $N$ we introduce on its right hand side an error of $R^{N+1}$. It is not straightforward to deduce what is the order of the error in $R$ of the scattered field inside the computational domain $\Omega$, i.e., with a constant which does not depend on $R$. The reason is that $R$ is not only a parameter in the asymptotic expansion (3.2) of the coefficient of the exact DtN boundary condition, but it is also the radius of $\Omega$. To overcome this problem we will study the error in the subdomain $\Omega_{0}$ of constant radius $R_{0}$ and with outer boundary $\Gamma_{0}$.

Note, that we cannot rely on the Lax-Milgram lemma and continuity and ellipticity constants of the bilinear form, which are independent of $R$, like in error analysis in [4] for the Poisson problem with Dirichlet boundary conditions on $\partial \Omega_{c}$. The idea of our proof, where as a key element the ABC is formulated on a a subdomain $\Omega_{0}$, may used to obtain rigorous proofs for other type of ABCs.

The normal derivative of the solution $u_{F, N}^{\mathrm{sc}}$ due to Feng's conditions on $\Gamma_{0}$ can be expressed using the representation (3.7) and $E_{n}^{N}(k, R)$ defined in (3.9) as

$$
\begin{aligned}
\left.\partial_{r} u_{F, N}^{\mathrm{sc}}(x)\right|_{\Gamma_{0}} & =\sum_{n \in \mathbb{Z}} k a_{n}^{N}\left(H^{(1)^{\prime}}\left(k R_{0}\right)-E_{n}^{N}(k, R) H_{n}^{(2) \prime}\left(k R_{0}\right)\right) \mathrm{e}^{\mathrm{i} n \theta(x)}, \\
& =\sum_{n \in \mathbb{Z}} \lambda_{n}^{N}\left(k, R_{0}, R\right) \mathrm{e}^{\mathrm{i} n \theta(x)} \widehat{u}_{F, N, n}^{\mathrm{sc}}\left(R_{0}\right)=:\left(\mathrm{F}_{N}\left(R_{0}, R\right) u_{F, N}^{\mathrm{sc}}\right)(x),
\end{aligned}
$$

where the coefficients of the DtN operator $\mathrm{F}_{N}\left(R_{0}, R\right)$

$$
\lambda_{n}^{N}\left(k, R_{0}, R\right):=k \frac{H_{n}^{(1) \prime}\left(k R_{0}\right)-E_{n}^{N}(k, R) H_{n}^{(2) \prime}\left(k R_{0}\right)}{H_{n}^{(1)}\left(k R_{0}\right)-E_{n}^{N}(k, R) H_{n}^{(2)}\left(k R_{0}\right)}=\lambda_{n}\left(k, R_{0}\right) \frac{1-E_{n}^{N}(k, R) \frac{H_{n}^{(2) \prime}\left(k R_{0}\right)}{H_{n}^{(1) \prime}\left(k R_{0}\right)}}{1-E_{n}^{N}(k, R) \frac{H_{n}^{(2)}\left(k R_{0}\right)}{H_{n}^{(1)}\left(k R_{0}\right)}}
$$


tends to the exact DtN coefficients $\lambda_{n}\left(k, R_{0}\right)$ if $E_{n}^{N}(k, R) \rightarrow 0$, hence, if $R \rightarrow \infty$. The solution $u_{F, N}^{\text {sc }}$ due to Feng's conditions on the outer boundary $\Gamma$ of the domain of radius $R$ can be written as solution of the same Helmholtz equation in $\Omega_{0}$ with the DtN boundary condition (3.11) on its outer boundary $\Gamma_{0}$ at $r=R_{0}$. The problem in $\Omega_{0}$ is related to a Fredholm operator.

Using the problem in $\Omega_{0}$ we can compare the solution for different radia $R$ of the computational domain $\Omega$ on the sub-domain $\Omega_{0}$. The dependence of the solution on $R$ is expressed in the DtN coefficients $\lambda_{n}^{N}\left(k, R_{0}, R\right)$.

We can write

$$
\begin{aligned}
\lambda_{n}\left(k, R_{0}\right)-\lambda_{n}^{N}\left(k, R_{0}, R\right) & =\lambda_{n}\left(k, R_{0}\right)\left(\begin{array}{c}
1-E_{n}^{N}(k, R) \frac{H_{n}^{(2) \prime}\left(k R_{0}\right)}{H_{n}^{(1) \prime}\left(k R_{0}\right)} \\
1-\frac{E_{n}^{N}(k, R) \frac{H_{n}^{(2)}\left(k R_{0}\right)}{H_{n}^{(1)}\left(k R_{0}\right)}}{1-H_{n}^{(2) \prime}\left(k R_{0}\right)}
\end{array}\right. \\
& =E_{n}^{N}(k, R) \lambda_{n}\left(k, R_{0}\right) \frac{\frac{H_{n}^{(2)}\left(k R_{0}\right)}{H_{n}^{(1)}\left(k R_{0}\right)}-\frac{H_{n}^{(1) \prime}\left(k R_{0}\right)}{H_{n}^{(2)}} .}{1-E_{n}^{N}(k, R) \frac{H_{n}^{(2)}\left(k R_{0}\right)}{H_{n}^{(1)}\left(k R_{0}\right)}} .
\end{aligned}
$$

Note, that due to $\left|H_{n}^{(2)}\left(k R_{0}\right)\right|=\left|H_{n}^{(1)}\left(k R_{0}\right)\right|$ the denominator in (3.13) cannot become zero if $\left|E_{n}^{N}(k, R)\right|<1$, which is fulfilled if $R$ is large enough, $E_{n}^{N}(k, R) \rightarrow 0$ for $R \rightarrow \infty$ as uniformly in $n$. The nominator in (3.13) can also not become zero, as $H_{n}^{(2)}\left(k R_{0}\right) H_{n}^{(1) \prime}\left(k R_{0}\right)-H_{n}^{(2) \prime}\left(k R_{0}\right) H_{n}^{(1)}\left(k R_{0}\right)=4 \mathrm{i} /\left(\pi R_{0}\right) \neq 0$ (see [1], 9.1.16, 9.1.27).

Hence, as the $E_{n}^{N}(k, R) \rightarrow 0$ for $R \rightarrow \infty$, we have in view of (3.10) asymptotically for $R \rightarrow \infty$ the estimate

$$
\left|\lambda_{n}\left(k, R_{0}\right)-\lambda_{n}^{N}\left(k, R_{0}, R\right)\right| \leq C_{N}\left(n, R_{0}\right) k(k R)^{-(N+1)},
$$

where the constants $C_{N}\left(n, R_{0}\right)$ do not depend on $R$ or $k$. The behaviour of $C_{N}\left(n, R_{0}\right)$ for $n \rightarrow \infty$, and so to follow the mapping property of the difference of the exact DtN operator $\mathrm{F}_{\infty}\left(R_{0}\right)$ and $\mathrm{F}_{N}\left(R_{0}, R\right)$ on $\Gamma_{0}$, is not straightforward to see. We will see, however, that these mapping property is not essential to prove the convergence of the modelling error. This is the main result of the error analysis, which we state in the following theorem. As we aim in explicit dependence of $R$, but not of $k$, all constants depend in the following on $k$ except where otherwise specified.

Theorem 3.1. Let $u_{F, N}:=u_{F, N}^{\mathrm{sc}}+u^{\mathrm{inc}}$ the unique solution of (2.1) in $\Omega$ with Feng's conditions of order $N$ on $\Gamma$ and let $e_{F, N}:=u_{F, N}-u=u_{F, N}^{\mathrm{sc}}-u^{\mathrm{sc}}$. Then, for any $R_{0}>R_{C}$ fixed and $S_{0}:=B_{R_{0}} \backslash \bar{B}_{R_{C}}$ it holds with a constant $C_{N}=C_{N}\left(k, R_{0}\right)$ independent of $R$ that

$$
\left\|e_{F, N}\right\|_{H^{1}\left(\Omega_{0}\right)} \leq C_{N} R^{-(N+1)}\left\|u^{\text {inc }}\right\|_{H^{1}\left(S_{0}\right)} .
$$

The constant $C_{N}$ is not bounded for $N \rightarrow \infty$ due to the fact that the local ABCs by Feng are based on an asymptotic expansion of the Hankel functions. This asymptotic expansion is not convergent for fixed $R$ and $N \rightarrow \infty$. Increasing the order from $N$ to $N+1$ results in a reduction of the modelling error only if the domain radius $R$ is large enough ( $c f$. the results of numerical experiments in Sect. 4).

For the proof of Theorem 3.1 we need the following.

Lemma 3.2. Let $u_{F, N}:=u_{F, N}^{\mathrm{sc}}+u^{\mathrm{inc}}$ the unique solution of (2.1) in $\Omega$ with Feng's conditions of order $N$ on $\Gamma$. Then, for any $R_{0}>R_{C}$ fixed it holds with a constant $C_{N}=C_{N}\left(k, R_{0}\right)$ independent of $R$ that

$$
\left\|\partial_{r} u_{F, N}^{\mathrm{sc}}\right\|_{H^{-1 / 2}\left(\Gamma_{0}\right)}+\left\|u_{F, N}^{\mathrm{sc}}\right\|_{H^{1 / 2}\left(\Gamma_{0}\right)} \leq C_{N}\left(R_{0}\right)\left\|u^{\mathrm{inc}}\right\|_{H^{1}\left(S_{0}\right)} .
$$


Proof of Theorem 3.1. It is easy to see that the exact solution $u$ is solution of the Helmholtz equation in $\Omega_{0}$ with exact DtN boundary conditions (3.1) on $r=R_{0}$, where $R$ is replaced by $R_{0}$,

$$
\left.\partial_{r} u^{\mathrm{sc}}(x)\right|_{\Gamma_{0}}=\sum_{n \in \mathbb{Z}} \lambda_{n}\left(k, R_{0}\right) \mathrm{e}^{\mathrm{i} n \theta} \widehat{u}_{n}^{\mathrm{sc}}\left(R_{0}\right)=:\left(\mathrm{F}_{\infty}\left(R_{0}\right) u^{\mathrm{sc}}\right)(x),
$$

and where $\widehat{u}_{n}^{\mathrm{sc}}\left(R_{0}\right)=\left(2 \pi R_{0}\right)^{-1} \int_{\Gamma_{0}} u^{\mathrm{sc}}(x) \mathrm{e}^{-\mathrm{i} n \theta(x)} \mathrm{d} \sigma(x)$. Here, we denote $\mathrm{F}_{\infty}\left(R_{0}\right)$ the exact DtN operator on $r=R_{0}$.

As $u_{F, N}$ is solution of the Helmholtz equation in $\Omega_{0}$ with (3.11) on $r=R_{0}$, the modelling error $e_{F, N}$ satisfies

$$
\begin{aligned}
-\Delta e_{F, N}(x)-\kappa^{2}(x) e_{F, N} & =0, \quad \text { in } \Omega_{0}, \\
\partial_{n} e_{F, N}(x) & =0, \quad \text { on } \partial \Omega_{0} \backslash \Gamma_{0}, \\
\left(\partial_{r} e_{F, N}\right)(x)-\left(\mathrm{F}_{\infty}\left(R_{0}\right) e_{F, N}\right)(x) & =\left(\mathrm{F}_{N}\left(R_{0}, R\right)-\mathrm{F}_{\infty}\left(R_{0}\right)\right) u_{F, N}^{\text {sc }} \quad \text { on } \Gamma_{0} .
\end{aligned}
$$

The system (3.16) is with exact DtN boundary conditions at $\Gamma_{0}$ and admits a unique solution. Since (3.16) is stated on a fixed domain $\Omega_{0}$ and only the left hand side of the boundary condition does not depend on $R$ we can assert

$$
\begin{aligned}
\left\|e_{F, N}\right\|_{H^{1}\left(\Omega_{0}\right)} & \left.\leq C_{N}\left(R_{0}\right) \|\left(\mathrm{F}_{N}\left(R_{0}, R\right)-\mathrm{F}_{\infty}\left(R_{0}\right)\right) u_{F, N}^{\mathrm{sc}}\right) \|_{H^{-1 / 2}\left(\Gamma_{0}\right)} \\
& \left.\leq C_{N}\left(R_{0}\right) \| \partial_{r} u_{F, N}^{\mathrm{sc}}-\mathrm{F}_{\infty}\left(R_{0}\right) u_{F, N}^{\mathrm{sc}}\right)\left\|_{H^{-1 / 2}\left(\Gamma_{0}\right)} \leq C_{N}\left(R_{0}\right)\right\| u^{\mathrm{inc}} \|_{H^{1}\left(S_{0}\right)} .
\end{aligned}
$$

where we used the definition of $\mathrm{F}_{N}\left(R_{0}, R\right)$, that $\mathrm{F}_{\infty}\left(R_{0}\right)$ is continuous from $H^{1 / 2}\left(\Gamma_{0}\right)$ to $H^{-1 / 2}\left(\Gamma_{0}\right)$, the triangle inequality and Lemma 3.2. In this estimate we applied $\mathrm{F}_{N}\left(R_{0}, R\right)-\mathrm{F}_{\infty}\left(R_{0}\right)$ only to the function $u_{F, N}^{\mathrm{sc}}$, which possess some higher regularity. The estimate is an upper bound in terms of $R$. As the coefficients $\lambda_{n}^{N}\left(k, R_{0}, R\right)-\lambda_{n}\left(k, R_{0}\right)$ of $\mathrm{F}_{N}\left(R_{0}, R\right)-\mathrm{F}_{\infty}\left(R_{0}\right)$ are bounded by $R^{-(N+1)}$, see (3.14), for any $n \in \mathbb{Z}$, the statement of the theorem follows.

It remains to show that the Dirichlet and Neumann traces of $u_{F, N}^{\mathrm{sc}}$ on $\Gamma_{0}$ are bounded independently of $R$.

Proof of Lemma 3.2. First, we are going to derive an alternative formulation in $\Omega$. In the original variational formulation (2.3) the incoming wave appears in an integral on $\Gamma$, whose locus depends on $R$. To obtain an estimate which is independent on $R$ we use an equivalent formulation for a linear combination $u_{F, N}^{\mathrm{a} u x}$ of the total and the scattered field. More precisely, we define

$$
u_{F, N}^{\mathrm{a} u x}(x):=u_{F, N}(x)-(1-\chi(x)) u^{\mathrm{inc}}(x)=u_{F, N}^{\mathrm{sc}}(x)+\chi(x) u^{\mathrm{inc}}(x),
$$

where $\chi(x)$ is a smooth and monotone cut-off function, which is 1 for $|x|<R_{C}+\delta$ and 0 for $|x|>R_{0}-\delta$ for some $\delta>0$. Hence, $u_{F, N}^{\text {aux }}(x)=u_{F, N}(x)$ for $|x|<R_{C}+\delta$, which fulfills the Helmholtz equation in $\Omega \cap B_{R_{C}+\delta}$. For $|x| \geq R_{C}+\delta$ the total, the incoming and the scattered field satisfy the Helmholt equation with $\kappa(x)=k$, and so $u_{F, N}^{\mathrm{a} u x}$. Furthermore, $u_{F, N}^{\mathrm{a} u x}(x)=u_{F, N}^{\mathrm{sc}}(x)$ for $r>R_{0}-\delta$, so Feng's condition on $\Gamma$ applies directly to $u_{F, N}^{\mathrm{a} u x}$.

Now, the variational formulation reads: Seek $u_{F, N}^{\text {aux }} \in V_{\lfloor N / 2\rfloor}$ such that

$$
\mathrm{a}_{\lfloor N / 2\rfloor}\left(u_{F, N}^{\mathrm{a} u x}, v\right)=\int_{S_{0}}\left(\nabla\left(\chi u^{\mathrm{inc}}\right) \cdot \nabla \bar{v}-k^{2} \chi u^{\mathrm{inc}} \bar{v}\right) \mathrm{d} \sigma(x), \quad \forall v \in V_{\lfloor N / 2\rfloor},
$$

where the coefficients in $a_{\lfloor N / 2\rfloor}$ are those from Feng's conditions. The variational formulation has a unique solution for $R$ large enough by Lemma 2.7. Now, by assuming $\nabla \chi \in L^{\infty}\left(S_{0}\right)$ we can assert that

$$
\left\|u_{F, N}^{\mathrm{aux}}\right\|_{H^{1}(\Omega)} \leq\left\|u_{F, N}^{\mathrm{aux}}\right\|_{V_{\lfloor N / 2\rfloor}} \leq C_{N}(R)\left\|u^{\mathrm{inc}}\right\|_{H^{1}\left(S_{0}\right)},
$$


where the constants $C_{N}(R)$ depend on $R$. In the neighbourhood of $\Gamma_{0}$ we have $u_{F, N}^{\mathrm{a} u x}=u_{F, N}^{\mathrm{sc}}$ and so using the trace theorems and the fact that $\Delta u_{F, N}^{\mathrm{a} u x}+k^{2} u_{F, N}^{\mathrm{a} u x}=0$ in $S_{0}$,

$$
\begin{aligned}
\left\|u_{F, N}^{\mathrm{sc}}\right\|_{H^{1 / 2}\left(\Gamma_{0}\right)} & \leq C\left(R_{0}\right)\left\|u_{F, N}^{\mathrm{a} u x}\right\|_{H^{1}\left(S_{0}\right)} \leq C_{N}\left(R, R_{0}\right)\left\|u^{\mathrm{inc}}\right\|_{H^{1}\left(S_{0}\right)}, \\
\left\|\partial_{r} u_{F, N}^{\mathrm{sc}}\right\|_{H^{-1 / 2}\left(\Gamma_{0}\right)} & \leq C\left(R_{0}\right)\left(\left\|u_{F, N}^{\mathrm{a} u x}\right\|_{H^{1}\left(S_{0}\right)}+\left\|\Delta u_{F, N}^{\mathrm{a} u x}\right\|_{L^{2}\left(S_{0}\right)}\right) \\
& =C\left(R_{0}\right)\left(\left\|u_{F, N}^{\mathrm{a} u x}\right\|_{H^{1}\left(S_{0}\right)}+k^{2}\left\|u_{F, N}^{\mathrm{a} u x}\right\|_{L^{2}\left(S_{0}\right)}\right) \\
& \leq C_{N}\left(R, R_{0}\right)\left\|u^{\mathrm{inc}}\right\|_{H^{1}\left(S_{0}\right)} .
\end{aligned}
$$

It remains to show that we can find constants $C_{N}\left(R_{0}\right)$ such that $C_{N}\left(R, R_{0}\right) \leq C_{N}\left(R_{0}\right)$. As the coefficients in the bilinear forms a $a_{\lfloor N / 2\rfloor}$ depend continuously on $R$ the same holds for $u_{F, N}^{\text {aux }}$ and so $C_{N}\left(R, R_{0}\right)$. Therefore, we have only to show that $C_{N}\left(R, R_{0}\right)$ tend to constants $C_{N, \infty}\left(R_{0}\right)$ for $R \rightarrow \infty$.

With $\lim _{R \rightarrow \infty} \lambda_{k}^{N}=\mathrm{i} k, \lim _{R \rightarrow \infty} E_{n}^{N}(k, R) \rightarrow 0$ we have $\lim _{R \rightarrow \infty} \lambda_{n}^{N}\left(k, R_{0}, R\right)=\lambda_{n}\left(k, R_{0}\right)$. Hence, $u_{F, N}^{\mathrm{sc}} \rightarrow$ $u^{\mathrm{sc}}$ and so $u_{F, N}^{\mathrm{a} u x} \rightarrow u^{\mathrm{aux}}:=u-(1-\chi) u^{\mathrm{inc}}$ which solves in $\Omega_{0}$ the variational formulation: Seek $u^{\text {aux }} \in H^{1}\left(\Omega_{0}\right)$ such that

$$
\begin{aligned}
& \int_{\Omega_{0}}\left(\nabla u^{\mathrm{a} u x} \cdot \nabla \bar{v}-\kappa^{2} u^{\mathrm{aux}} \bar{v}\right) \mathrm{d} x-\sum_{n \in \mathbb{Z}} \lambda_{n}\left(k, R_{0}\right) \int_{\Gamma_{0}} u^{\mathrm{a} u x} \mathrm{e}^{-\mathrm{i} n \theta(x)} \mathrm{d} \sigma(x) \int_{\Gamma_{0}} \bar{v} \mathrm{e}^{\mathrm{i} n \theta(x)} \mathrm{d} \sigma(x) \\
& =\int_{S_{0}}\left(\nabla\left(\chi u^{\mathrm{inc}}\right) \cdot \nabla \bar{v}-k^{2} \chi u^{\mathrm{inc}} \bar{v}\right) \mathrm{d} \sigma(x), \quad \forall v \in H^{1}\left(\Omega_{0}\right) .
\end{aligned}
$$

This problem stated in a fixed domain with exact DtN boundary conditions is well-posed. So, we have with constants $C\left(R_{0}\right)$ independent of $R$ that

$$
\left\|u^{\mathrm{aux}}\right\|_{H^{1}\left(\Omega_{0}\right)} \leq C\left(R_{0}\right)\left\|u^{\mathrm{inc}}\right\|_{H^{1}\left(S_{0}\right)} .
$$

We can bound $\left\|u^{\mathrm{sc}}\right\|_{H^{1 / 2}\left(\Gamma_{0}\right)}$ and $\left\|\partial_{r} u^{\mathrm{sc}}\right\|_{H^{-1 / 2}\left(\Gamma_{0}\right)}$ by $\left\|u^{\mathrm{a} u x}\right\|_{H^{1}\left(\Omega_{0}\right)}$, which implies the existence of $C_{N, \infty}\left(R_{0}\right)$ as limit of $C_{N}\left(R, R_{0}\right)$ for $R \rightarrow \infty$.

This finishes the proof.

\section{NuMERICAL EXPERIMENTS}

We have implemented the discontinuous Galerkin formulation introduced in Schmidt, Diaz and Heier [27] for the Feng- 4 and Feng-5 conditions in the numerical C++ library Concepts $[8,14,28]$, as well as Feng-0 till Feng-3 with the usual continuous formulations (compare [31] for implementational details related to BGT absorbing boundary conditions). The $h p$-FEM part of Concepts is based on quadrilateral, curved cells in 2D where the polynomial degree can be set independently in each cell and even anisotropically. With cells having circular edges the circular boundary can be exactly resolved (see Fig. 2), where a geometry error appears only in the numerical quadrature of the integrals.

For the numerical experiments we study two model problems:

A. the acoustic scattering on a rigid cylinder with circular cross-section, where the computational domain $\Omega$ is the disk of radius $R$ without the disk of radius $R_{D}=1$ (see Fig. 1a) and $k=1$, and

B. the electromagnetic scattering on two dielectric cylinders, whose cross-section are equilateral triangles of length $a=1.05$ and distance $d=0.25$ (see [22] and Fig. 1b). We have $\kappa^{2}(x)=\varepsilon(x) \omega^{2}$ with the angular frequency $\omega$ and the (relative) dielectricity $\varepsilon(x)$, which is $-40.2741+2.794 \mathrm{i}$ inside the cylinders and 1 outside, hence $k=\omega$. We choose as frequency $\omega=0.638$ corresponding to a wave-length in the exterior $\lambda=2 \pi / k=9.84$.

For both model problems the incident wave is a plane wave in direction $(1,0)^{\top}$ (from left). For model problem $\mathrm{B}$ the quadrilateral mesh is generated with gmsh $2.6 .2[15,25]$ where we use a local refinement close to the nodes 


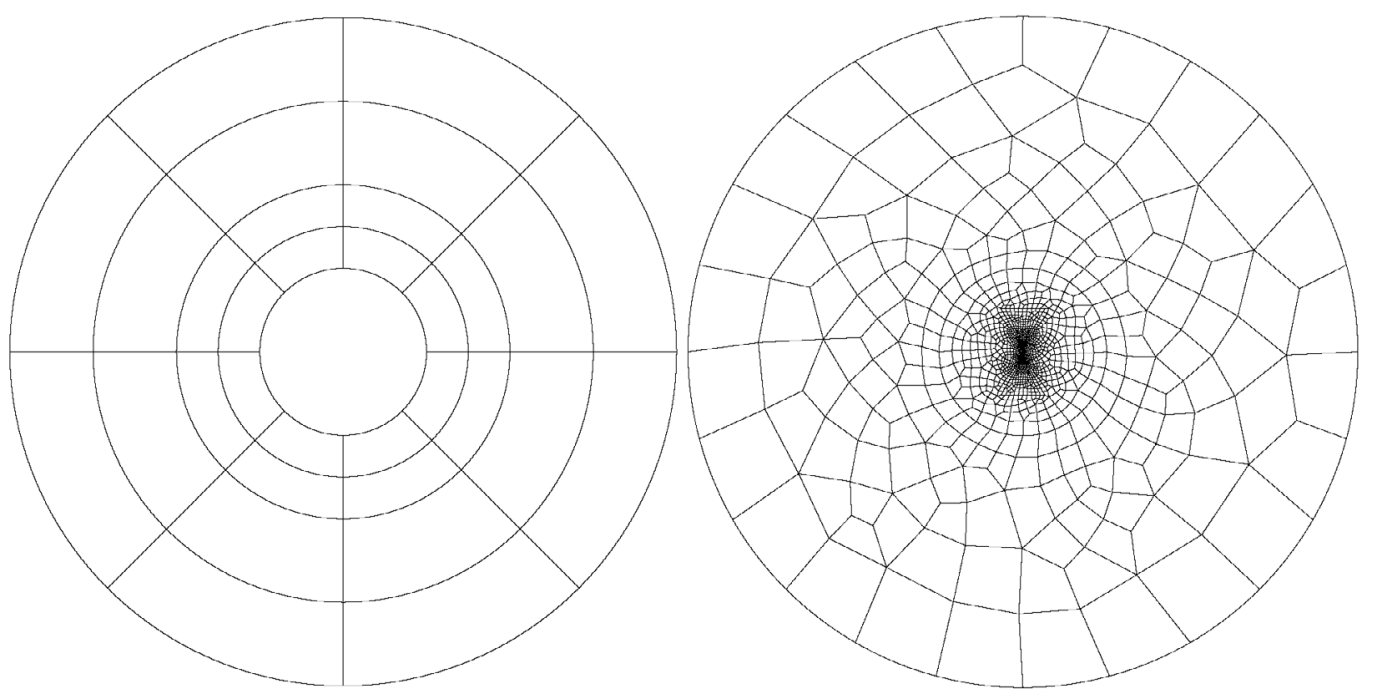

Figure 2. Curvilinear, quadrilateral meshes for the scattering on a circular disk (model problem A.) and on plasmonic triangles (model problem B.) in Concepts.

of the triangles (see Fig. 2). We computed for both problems the error in a fixed domain $\Omega_{0}:=\Omega \cap B_{R_{0}}$ for some $R_{0}$, which we will specify for each experiment.

First we study the modelling error of Feng's conditions up to order 5 on the model problem A. For this we use a coarse mesh with a uniform polynomial degree up to 20 for the largest domain radius, such that a discretisation error is negligible to the modelling error. An approximation to the exact solution is obtained by using the truncated exact DtN boundary conditions with up to 20 terms and a polynomial degree up to 20 such that both, the modelling and the discretisation error are negligible to the those of the experiments with Feng's conditions. While changing the domain radius $R$ the error is computed on the fixed domain $\Omega_{0}$ with $R_{0}=2$. The results of the convergence analysis for Feng's conditions are shown in Figure 3. We obtain convergence orders 1.0 up to 6.0 for Feng-0 up to Feng-5, which confirms that the estimates of the modelling error in $R$ given in Theorem 3.1 are sharp.

Using absorbing boundary conditions of higher order implies a reduction of the modelling error for a given domain $\Omega$, if its outer radius $R$ is large enough. On the other hand, to obtain a given error level smaller domains can be used. We have computed the solution of the model problem B with different domain radia $R$, where we were searching for the radius $R$ necessary to obtain a relative $L^{2}\left(\Omega_{0}\right)$-error of $10^{-2}, 10^{-4}$ or $10^{-6}$, respectively, with a tolerance of $10 \%$. If we are not able to obtain the error levels, that is, if the computations with a domain with the necessary radius would be to expensive, we have extrapolated the computed error levels. The results of this study are shown in Table 3. For a relative $L^{2}\left(\Omega_{0}\right)$-modelling error of about $10^{-2}$ Feng's conditions of order 1 (equivalent to the BGT-1 condition) need with $R=3.80$ a over 14 times smaller domain radius than Feng-0. The use of Feng-2 reduce the radius to 2.65 where a further increase of the order $N$ does not lead to smaller radia. These results illustrate Feng's conditions are based on an asymptotic expansion in $R$ rather than a convergent series in $N$ as stated in Theorem 3.1.

For relative $L^{2}\left(\Omega_{0}\right)$-modelling error of about $10^{-4}$ and $10^{-6}$ increasing the order in Feng's conditions lead to smaller radia. Using Feng-5 with its additional second tangential derivatives in the variational formulation instead of Feng-3 leads to a reduction of the radius from 28.2 to 14.1 for the relative error level of $10^{-6}$, and in comparison to Feng-2 the needed radius is more than 4 times smaller. 


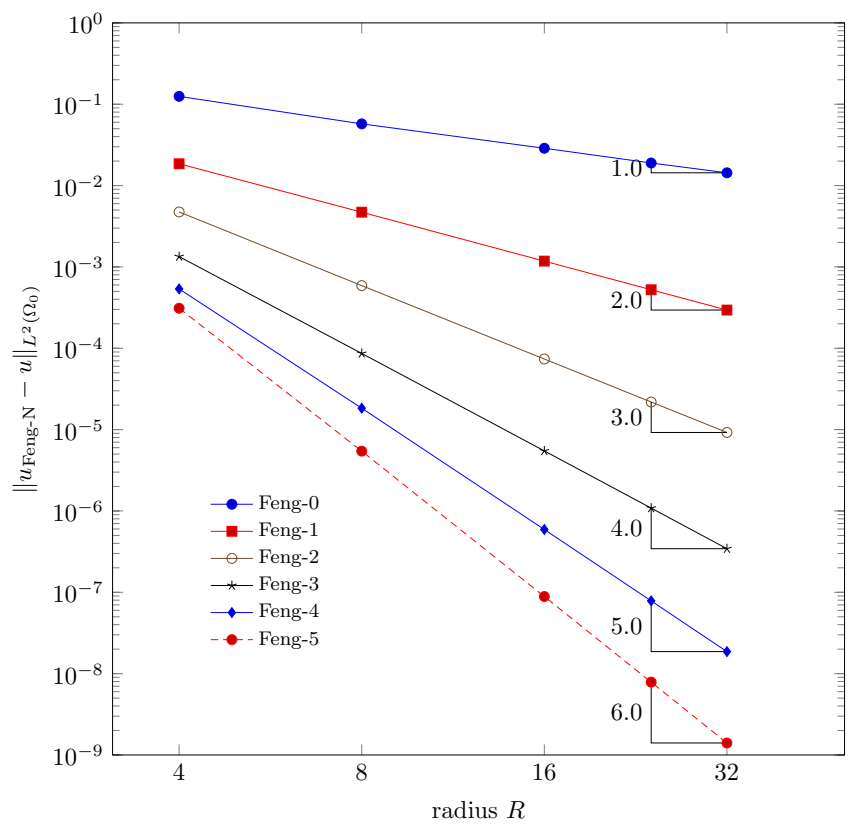

FIgURE 3. Convergence of the modelling error of the absorbing boundary conditions Feng-N for $N=0, \ldots, 5$ with respect to the radius $R$ for $R=4,8,16,24,32$ for the scattering on a circular disk of radius $R_{D}=1$. The polynomial order of the finite element discretisation is chosen that high that the discretisation is negligible.

TABLE 3 . The radia of the computational domains which are necessary to obtain given relative $L^{2}\left(\Omega_{0}\right)$-norm error levels $( \pm 10 \%)$ for Feng-N for $N=0, \ldots, 5$ for the model problem B of electromagnetic scattering on two dielectric cylinders of tringular cross-section (see Fig. 1b). The values with a star are extrapolated.

\begin{tabular}{cccccc}
\hline \multicolumn{2}{l}{ modelling error $10^{-2}$} & \multicolumn{2}{l}{ modelling error $10^{-4}$} & \multicolumn{2}{l}{ modelling error $10^{-6}$} \\
\hline $\mathrm{N}$ & $\mathrm{R}$ & $\mathrm{N}$ & $\mathrm{R}$ & $\mathrm{N}$ & $\mathrm{R}$ \\
\hline 0 & $55.27^{*}$ & 0 & $5527^{*}$ & 0 & $552747^{*}$ \\
1 & 3.80 & 1 & $39.0^{*}$ & 1 & $392^{*}$ \\
2 & 2.65 & 2 & 13.6 & 2 & $61.0^{*}$ \\
3 & 2.50 & 3 & 8.65 & 3 & 28.2 \\
4 & 2.60 & 4 & 7.10 & 4 & 18.2 \\
5 & 2.80 & 5 & 6.30 & 5 & 14.1 \\
\hline
\end{tabular}

\section{Conclusion}

We analysed symmetric local absorbing boundary conditions for the Helmholtz equation with respect to their well-posedness. There might be spurious eigenmodes, which vanish for Feng's conditions if the radius of the truncated domain is chosen to be large enough. We analysed the modelling error in a fixed subdomain $\Omega_{0}$ in dependence of the radius of the truncated domain, on which outer boundary the conditions are applied. For this we formulated non-local Dirichlet-to-Neumann boundary conditions on the outer boundary of $\Omega_{0}$ such that the solution coincides with that using Feng's conditions on the outer boundary of $\Omega$. Comparing this Dirichlet-toNeumann map with the Dirichlet-to-Neumann map related to the (exact) radiation condition by Sommerfeld, 
we showed that the modelling error behaves like $R^{-(N+1)}$ for Feng's conditions of order $N$. Our proofs confirm that the approximation of the exact Dirichlet-to-Neumann boundary conditions up to $R^{-(N+1)}$ transfer to the modelling error in a fixed subdomain. The ideas of the proofs might be applied to the more involved BGT conditions, both in two and three dimensions.

\section{Appendix A.}

Proof of Lemma 2.7. We consider the eigenproblem (2.3) (for $k$ fixed) and $f_{J}=0$ for varying domain radia $R$. Then, we have to show that the only solution for $R \rightarrow \infty$ is the trivial solution $u=0$.

To this end we act by contradiction: Assume that there exists a sequence $\left\{R_{\ell}\right\}_{\ell=1}^{\infty}$ with $R_{\ell+1}>R_{\ell}$ and $R_{\ell} \rightarrow \infty$ for $\ell \rightarrow \infty$ such that $u_{\ell} \in V_{J}$ is a non-trivial solution of (2.3) with $f_{J}=0$ in the domain $\Omega=\Omega_{\ell}$ with radius $R=R_{\ell}$. Then, for $r>R_{0}$ we can write $u=u_{\ell}$ as

$$
u(x)=\sum_{n \in \mathbb{Z}}\left[b_{n}^{+}(R) H_{n}^{(1)}(k r)+b_{n}^{-}(R) H_{n}^{(2)}(k r)\right] \mathrm{e}^{\mathrm{i} n \theta},
$$

where the coefficients $b_{n}^{+}(R)$ correspond to the outgoing and $b_{n}^{-}(R)$ to the incoming waves. In this case of varying domain radius we prefer to use as normalisation of the eigenfunctions the condition

$$
-\left\langle\operatorname{Im}\left(\kappa^{2}\right) u, u\right\rangle+\sum_{j=0}^{\lfloor J\rfloor} \sum_{n \in \mathbb{Z}} n^{2 j}\left[\left|b_{n}^{+}(R)\right|^{2}+\left|b_{n}^{-}(R)\right|^{2}\right]=1,
$$

which does not depend in its evaluation on $R\left(\operatorname{as~} \operatorname{supp}\left(\kappa^{2}\right) \subset \Omega_{0}\right)$, but only through the $R$ dependent eigensolution. Due to the assumption on $\kappa$ all terms on the left hand side of (A.1) and we can assert the following uniform bounds

$$
\left|\left\langle\operatorname{Im}\left(\kappa^{2}\right) u, u\right\rangle\right| \leq 1, \quad \sum_{n \in \mathbb{Z}} n^{2 j}\left|b_{n}^{ \pm}(R)\right|^{2} \leq 1,
$$

in $R$. As by assumption on $a_{j}$ the conditions approach Feng's conditions of order 0 for $R \rightarrow \infty$ we obtain that $b_{n}^{-}(R) \rightarrow 0$ for $R \rightarrow \infty$. Hence, in view of the behaviour of $H_{n}^{(1)}(k R)$ for large arguments ([21], Chap. 1) we can write for $j=0, \ldots, J$

$$
R^{j+\frac{1}{2}} \mathrm{e}^{-\mathrm{i} k R} \partial_{\tau}^{j} u(x) \rightarrow \sqrt{\frac{\pi k}{2}} \sum_{n \in \mathbb{Z}}(\mathrm{i} n)^{j} b_{n}^{+}(R) \mathrm{e}^{-\mathrm{i}\left(\frac{n}{2}+\frac{1}{4}\right) \pi} \mathrm{e}^{\mathrm{i} n \theta} \text { on } \Gamma \text { for } R \rightarrow \infty .
$$

Note, that $b_{n}^{+}(R)=b_{n}^{-}(R)=0$ for all $n \in \mathbb{Z}$ implies $u=0$ in $\Omega$ due to the unique continuation principle ([23], Chap. 4.3). Hence, out assumption of the existence of a non-trivial solution $u$ were wrong if and only if $b_{n}^{+}(R) \rightarrow 0$ for $R \rightarrow \infty$.

Now, for $v=u$ taking the imaginary part of (2.3), multiplied by $1 /\left(\pi^{2} k R\right)$, we find that

$$
0=\lim _{R \rightarrow \infty}\left[-\frac{1}{\pi^{2} k R}\left\langle\operatorname{Im}\left(\kappa^{2}\right) u, u\right\rangle+\operatorname{Im} a_{0} \sum_{n \in \mathbb{Z}}\left|b_{n}^{+}(R)\right|^{2}+\sum_{j=1}^{J} \frac{\operatorname{Im} a_{j}}{R^{2 j}} \sum_{n \in \mathbb{Z}} n^{2 j}\left|b_{n}^{+}(R)\right|^{2}\right]=-k \lim _{R \rightarrow \infty} \sum_{n \in \mathbb{Z}}\left|b_{n}^{+}(R)\right|^{2},
$$

where we used the fact that the terms $\left|\left\langle\operatorname{Im}\left(\kappa^{2}\right) u, u\right\rangle\right|$ and $\sum_{n \in \mathbb{Z}} n^{2 j}\left|b_{n}^{+}(R)\right|^{2}$ are bounded by 1 , see (A.2). The equality (A.3) is only true if $b_{n}^{+}(R) \rightarrow 0$ for all $n \in \mathbb{Z}$. Hence, our assumption was wrong, and there is only the trivial solution for $R \rightarrow \infty$. This completes the proof. 


\section{REFERENCES}

[1] M. Abramowitz and I.A. Stegun, Handbook of Mathematical Functions with Formulas, Graphs, and Mathematical Tables. ninth dover printing, tenth gpo printing ed. Dover, New York (1964).

[2] R.A. Adams, Sobolev spaces. Academic Press, New York, London (1975).

[3] W. Arendt, G. Metafune, D. Pallara and S. Romanell, The Laplacian with Wentzell-Robin boundary conditions on spaces of continuous functions. Semigroup Forum 67 (2003) 247-261.

[4] W. Bao and H. Han, High-order local artificial boundary conditions for problems in unbounded domains. Comput. Methods Appl. Mech. Engrg. 188 (2000) 455-471.

[5] A. Bayliss, M. Gunzburger and E. Turkel, Boundary conditions for the numerical solution of elliptic equations in exterior regions. SIAM J. Appl. Math. 42 (1982) 430-451.

[6] V. Bonnaillie-Nol, M. Dambrine, F. Hrau and G. Vial, On generalized Ventcel's type boundary conditions for Laplace operator in a bounded domain. SIAM J. Math. Anal. 42 (2010) 931-945.

[7] D. Braess, Finite Elements: Theory, Fast Solvers, and Applications in Solid Mechanics, 3th eds. Cambridge University Press (2007).

[8] Concepts Development Team. Webpage of Numerical C++ Library Concepts 2. http://www.concepts.math.ethz.ch (2014).

[9] K.-J. Engel, The Laplacian on $C(\bar{\omega})$ with generalized Wentzell boundary conditions. Arch. Math. (Basel) 81 (2003) 548-558.

[10] B. Engquist and A. Majda, Absorbing boundary conditions for numerical simulation of waves. Proc. Nat. Acad. Sci. USA 74 (1977) 1765.

[11] W. Feller, The parabolic differential equations and the associated semi-groups of transformations. Ann. Math. 55 (1952) 468-519.

[12] W. Feller, Generalized second order differential operators and their lateral conditions. Illinois J. Math. 1 (1957) 459-504.

[13] K. Feng, Finite element method and natural boundary reduction. In Proc. of the International Congress of Mathematicians (1983) 1439-1453.

[14] P. Frauenfelder and C. Lage, Concepts - An Object-Oriented Software Package for Partial Differential Equations. Math. Model. Numer. Anal. 36 (2002) 937-951.

[15] C. Geuzaine and J.-F. Remacle, Gmsh: A 3-d finite element mesh generator with built-in pre-and post-processing facilities. Int. J. Numer. Methods Engrg. 79 (2009) 1309-1331.

[16] D. Givoli, Non-reflecting boundary conditions. J. Comput. Phys. 94 (1991) 1-29.

[17] D. Givoli, Numerical methods for problems in infinite domains. Elsevier, Amsterdam New York (1992).

[18] D. Givoli and J.B. Keller, Special finite elements for use with high-order boundary conditions. Comput. Methods Appl. Mech. Engrg. 119 (1994) 199-213.

[19] D. Givoli, I. Patlashenko and J.B. Keller, High-order boundary conditions and finite elements for infinite domains. Comput. Methods Appl. Mech. Engrg. 143 (1997) 13-39.

[20] H. Han and X. Wu, A survey on artificial boundary method. Sci. China Math. 56 (2013) 2439-2488.

[21] F. Ihlenburg, Finite Element Analysis of Acoustic Scattering. Springer-Verlag (1998).

[22] A.L. Koh, A.I. Fernández-Domínguez, D.W. McComb, S.A. Maier and J.K. Yang, High-resolution mapping of electron-beamexcited plasmon modes in lithographically defined gold nanostructures. Nano letters 11 (2011) 1323-1330.

[23] R. Leis, Initial Boundary Value Problems in Mathematical Physics. B. G. Teubner Gmbh (1986).

[24] W. McLean, Strongly Elliptic Systems and Boundary Integral Equations. Cambridge University Press (2000).

[25] J.-F. Remacle, J. Lambrechts, B. Seny, E. Marchandise, A. Johnen and C. Geuzainet, Blossom-quad: A non-uniform quadrilateral mesh generator using a minimum-cost perfect-matching algorithm. Int. J. Numer. Methods Engrg. 89 (2012) $1102-1119$.

[26] S. Sauter and C. Schwab, Boundary element methods. Springer-Verlag, Heidelberg (2011).

[27] K. Schmidt, J. Diaz and C. Heier, Non-conforming Galerkin finite element methods for symmetric local absorbing boundary conditions. In preparation.

[28] K. Schmidt and P. Kauf, Computation of the band structure of two-dimensional photonic crystals with $h p$ finite elements. Comput. Methods Appl. Mech. Engr. 198 (2009) 1249-1259.

[29] A. Venttsel', On boundary conditions for multidimensional diffusion processes. Theory Probab. Appl. 4 (1959) $164-177$.

[30] A.D. Venttsel', Semigroups of operators that correspond to a generalized differential operator of second order. Dokl. Akad. Nauk SSSR (N.S.) 111 (1956) 269-272.

[31] M. Wang, C. Engström, K. Schmidt and C. Hafner, On high-order FEM applied to canonical scattering problems in plasmonics. J. Comput. Theor. Nanosci. 8 (2011) 1-9. 